\title{
Inlfuence of Brain-Based Learning Stratgies on Academic Motivation, Stress and Self-Esteem of High School Students in North Banagalore
}

\author{
Reshma HS', Dr Smitha M Reddy ${ }^{2}$ \\ ${ }^{1}$ Research Scholar,Gardern City University \\ ${ }^{2}$ Research Supervisor,Assistant Professor,Garden City University
}

\begin{abstract}
Brain-Based Learning Strategy stimulates the whole brain for effective function which results in greater academic progress. This being the case it is bound to result in academic motivation, removal of stress and an increase in the self-esteem of students without any doubt.

Brain-Based Learning Strategy provides a safe and threat-free environment whereby the meaningful presentation of content prepares the learners' brains to store, process and retrieve the information in a soothing way. The main objective of this paper is to study the influence of brain-based learning strategies on the academic motivation, stress and self-esteem of high school students in North Bangalore, identity the factors of brain-based learning which influence learning process among high school students and then move on to identifying the factors of motivation, stress and self-esteem which influence the academic performance of high school students with respect to brain-based learning. The results of the study have confirmed that brain-based learning would result in motivation, removal of stress and higher self-esteem thereby resulting in improved academic performance.
\end{abstract}

Keywords

Brain-based learning strategy, motivation, stress, self-esteem, academic performance.

Article Received: 10 August 2020, Revised: 25 October 2020, Accepted: 18 November 2020

\section{Introduction}

Brain-Based Learning is acknowledged as an innovative strategy that could be adopted by schools which is learnercentric with the teacher facilitation the process. This undoubtedly would help in fully activating the cognitive skills of the learner resulting in a meaning learning experience with higher levels of clarity and understanding and longer retention.

Brain-Based Learning Strategy provides a safe and threatfree environment whereby the meaningful presentation of content prepares the learners' brains to store, process and retrieve the information in a soothing way (Aziz-ur-Rehman et al., 2012). Brain-based research suggests that when students explain what they have learned, they use the whole brain to do it, which makes long term storage and retrieval of information more efficiently (Wilson, 2004; Chavhan, 2012).

Brain-Based Learning Strategy stimulates the whole brain for effective function which results in greater academic progress, including reading comprehension, oral reading fluency, spelling, creative writing, math computation, problem solving, memory, accuracy focus and attention and higher scores of test results. This being the case it is bound to result in academic motivation, removal of stress and an increase in the self-esteem of students without any doubt.

\section{Review of Literature}

According to Keleş \& Çepni (2006). The main focus of brain-based learning is to ensure meaningful learning. This would require mapping which means new knowledge is linked with the previous knowledge in order to ensure meaningful learning.

Hasra (2007) has elaborated on the need to follow a phrased brain-based learning process which would help in making the learning more effective and permanent. The phrases include relaxed alertness, orchestrated immersion and active processing.

Zaudeh and Al Astal (2014) investigated the effectiveness of brain-based learning strategies profile on the multiple intelligences of children living under stressful conditions in Gaza. A case control study was conducted with a purposive sample of 93 students, 45 as experimental group and 48 as control group. The post test results indicated significant differences in all domains except in musical and intrapersonal intelligences. Brain-based learning strategies are effective and useful for linguistic, mathematical, spatial, kinaesthetic, interpersonal and naturalist intelligences.

The main advantage of the orchestrated immersion is that it helps the creating an image of the gist of the subject in a meaningful and vivid manner in the learners' minds. It is believed that if the learner is capable of grasping the gist through various sense organs, retention level of the new inputs would be much better. Materna (2000) strongly believes that this phase helps learners establish patterns and associations in their brains while providing them with rich and complex experiences in making learning more permanent.

\section{Objectives of the Research}

- To study the influence of brain-based learning strategies on the academic motivation, stress and self-esteem of high school students in North Bangalore.

- To identity the factors of brain-based learning which influence learning process among high school students

- To identity the factors of motivation, stress and self-esteem which influence the academic performance of high school students with respect to brain-based learning. 


\section{Research Methodology}

Descriptive research design was adopted for this study. Standardised measuring instruments which have been validated in testing influence of brain-based learning strategies on academic performance, stress and self-esteem (which are readily available) which includes the SMTBL questionnaire were used. The questionnaire was tested for internal consistency and the same was found to be high Cronbach's alpha estimated to be $\alpha=0.88$. Exploratory Factor Analysis (EFA) was used to check for the construct validity of the instrument. subscale.

The sampling framework included high school students in north Bangalore the sampling technique was convenience sampling and the sample size was 383 students drawn from 15 schools.

\section{Analysis}

\section{HYPOTHESIS I}

Null Hypothesis: There is no significant difference between gender and level of agreement

on influence of brain-based learning strategies on academic motivation, stress and self-esteem

Table $1: \mathrm{t}$ test for significant difference gender and level of agreement on influence of brain-based learning strategies on academic motivation, stress and self-esteem

\begin{tabular}{|c|c|c|c|c|c|c|}
\hline \multirow{3}{*}{ Factors } & \multicolumn{4}{|c|}{ Gender } & \multirow{3}{*}{$\begin{array}{c}\text { t } \\
\text { value }\end{array}$} & \multirow{3}{*}{$\begin{array}{c}P \\
\text { value }\end{array}$} \\
\hline & \multicolumn{2}{|c|}{ Male } & \multicolumn{2}{|c|}{ Female } & & \\
\hline & Mean & SD & Mean & SD & & \\
\hline $\begin{array}{c}\text { Brain based } \\
\text { learning } \\
\text { strategies }\end{array}$ & 2.68 & 4.53 & 25.40 & 2.68 & 420 & $0.015^{*}$ \\
\hline $\begin{array}{c}\text { Learning } \\
\text { value }\end{array}$ & 12.89 & 3.14 & 14.64 & 159 & 0.29 & $0.023^{*}$ \\
\hline $\begin{array}{c}\text { Active } \\
\text { learning } \\
\text { strategies } \\
\end{array}$ & 15.12 & 3.25 & 15.80 & 1.96 & .031 & $0.014 *$ \\
\hline Self-efficacy & 18.52 & 3.50 & 19.28 & 2.70 & 1.069 & $0.046^{*}$ \\
\hline $\begin{array}{l}\text { Performance } \\
\text { goal }\end{array}$ & 17.80 & 3.03 & 19.48 & 4.06 & 1.022 & $0.018^{*}$ \\
\hline $\begin{array}{l}\text { Achievement } \\
\text { goal }\end{array}$ & 10.47 & 4.35 & 12.44 & 2.86 & .169 & $0.024 *$ \\
\hline $\begin{array}{r}\text { Lear } \\
\text { enviro }\end{array}$ & 13.34 & 2.89 & 12.32 & 1.11 & 1.751 & $0.031 *$ \\
\hline $\begin{array}{c}\text { Overall } \\
\text { student } \\
\text { motivation }\end{array}$ & 15.38 & 2.70 & 13.04 & 3.09 & 4.170 & $0.034 *$ \\
\hline Emotional & 9.19 & 4.22 & 20.80 & 5.05 & 2.637 & $0.019 *$ \\
\hline Physiologic & 23.11 & 4.45 & 23.76 & 2.37 & .039 & $0.039 *$ \\
\hline Behaviour & 16.75 & 4.21 & 24.28 & 2.85 & 1.973 & $0.049 *$ \\
\hline Cognitive & 22.11 & 4.38 & 29.16 & 4.20 & 1.394 & $0.048^{*}$ \\
\hline $\begin{array}{c}\text { Overall } \\
\text { academic } \\
\text { stress } \\
\end{array}$ & 22.64 & 5.40 & 25.28 & 4.89 & 1.224 & $0.022 *$ \\
\hline Family & 19.30 & 4.76 & 20.48 & 4.31 & 1.371 & $0.017^{*}$ \\
\hline School & 14.24 & 2.98 & 15.40 & 2.74 & 6.273 & $0.021 *$ \\
\hline
\end{tabular}

\begin{tabular}{|c|c|c|c|c|c|c|}
\hline Peer & 15.86 & 3.54 & 16.64 & 4.94 & 2.962 & $0.034^{*}$ \\
\hline $\begin{array}{c}\text { Overall } \\
\text { student self- } \\
\text { esteem }\end{array}$ & 17.27 & 3.47 & 18.60 & 4.64 & .512 & $0.023^{*}$ \\
\hline Overall & 20.13 & 4.68 & 22.24 & 4.37 & 1.453 & $0.047^{*}$ \\
\hline
\end{tabular}

Since $\mathrm{P}$ value is less than 0.05 , null hypothesis is rejected at $5 \%$ level with respect to all the above factors of brain-based learning strategies influencing academic motivation, stress and self-esteem among high school students. Hence there is significant difference between gender and level of agreement on factors of brain-based learning strategies influencing academic motivation, stress and self-esteem among high school students. Based on mean score, it can be seen that females have indicated a higher level of agreement with respect to all the factors of brain-based learning strategies influencing academic motivation, stress and self-esteem among high school students.

\section{HYPOTHESIS II}

Null Hypothesis: There is no significant difference between age and level of agreement on influence of brain-based learning strategies on academic motivation, stress and self-esteem

Table 2: $t$ test for significant difference age and level of agreement on influence of brain-based learning strategies on academic motivation, stress and self-esteem

\begin{tabular}{|c|c|c|c|c|c|c|}
\hline \multirow[b]{3}{*}{ Factors } & \multicolumn{4}{|c|}{ Age of students } & \multirow{3}{*}{$\begin{array}{c}\mathbf{t} \\
\text { valu } \\
\mathbf{e}\end{array}$} & \multirow{3}{*}{$\begin{array}{c}\mathrm{P} \\
\text { value }\end{array}$} \\
\hline & \multicolumn{2}{|c|}{$<16$} & \multicolumn{2}{|c|}{$>16$} & & \\
\hline & $\begin{array}{c}\text { Mea } \\
\mathrm{n}\end{array}$ & SD & $\begin{array}{c}\text { Mea } \\
n\end{array}$ & $\mathrm{SD}$ & & \\
\hline $\begin{array}{c}\text { Brain based } \\
\text { learning } \\
\text { strategies }\end{array}$ & 31.58 & 4.94 & 33.61 & $\begin{array}{c}5.0 \\
6\end{array}$ & 2.185 & $\begin{array}{c}0.029 \\
*\end{array}$ \\
\hline $\begin{array}{l}\text { Learning } \\
\text { value }\end{array}$ & 15.72 & 3.40 & 16.88 & $\begin{array}{c}3.0 \\
6 \\
\end{array}$ & 0.487 & $\begin{array}{c}0.027 \\
* \\
\end{array}$ \\
\hline $\begin{array}{c}\text { Active } \\
\text { learning } \\
\text { strategies }\end{array}$ & 17.12 & 3.73 & 18.23 & $\begin{array}{c}3.2 \\
6\end{array}$ & 2.583 & $\begin{array}{c}0.030 \\
*\end{array}$ \\
\hline Self-efficacy & 17.82 & $\begin{array}{c}13.5 \\
2\end{array}$ & 18.04 & $\begin{array}{c}2.2 \\
6\end{array}$ & 1.176 & $\begin{array}{c}0.021 \\
* \\
\end{array}$ \\
\hline $\begin{array}{c}\text { Performance } \\
\text { goal }\end{array}$ & 20.17 & 4.67 & 20.55 & $\begin{array}{c}4.0 \\
3\end{array}$ & 0.887 & $\begin{array}{c}0.037 \\
*\end{array}$ \\
\hline $\begin{array}{c}\text { Achievemen } \\
\text { t goal }\end{array}$ & 13.03 & 2.90 & 13.43 & $\begin{array}{c}2.7 \\
7\end{array}$ & 1.416 & $\begin{array}{c}0.049 \\
*\end{array}$ \\
\hline $\begin{array}{c}\text { Learning } \\
\text { environment }\end{array}$ & 14.94 & 3.02 & 15.43 & $\begin{array}{c}2.6 \\
1 \\
\end{array}$ & 1.759 & $\begin{array}{c}0.041 \\
* \\
\end{array}$ \\
\hline $\begin{array}{c}\text { Overall } \\
\text { student } \\
\text { motivation } \\
\end{array}$ & 16.13 & 3.27 & 19.41 & $\begin{array}{c}3.3 \\
4\end{array}$ & 1.560 & $\begin{array}{c}\mathbf{0 . 0 3 4} \\
*\end{array}$ \\
\hline Emotional & 13.34 & 4.62 & 14.09 & $\begin{array}{c}4.0 \\
7 \\
\end{array}$ & 1.179 & $\begin{array}{c}0.039 \\
* \\
\end{array}$ \\
\hline Physiologic & 15.44 & 4.76 & 17.30 & $\begin{array}{c}2.5 \\
4\end{array}$ & 3.056 & $\begin{array}{c}0.002 \\
*\end{array}$ \\
\hline Behaviour & 10.33 & 3.48 & 12.90 & $\begin{array}{c}2.0 \\
5\end{array}$ & 2.503 & 0.013 \\
\hline Cognitive & 16.59 & 2.49 & 17.53 & $\begin{array}{c}2.3 \\
1\end{array}$ & 0.119 & $\begin{array}{c}0.029 \\
*\end{array}$ \\
\hline
\end{tabular}




\begin{tabular}{|c|c|c|c|c|c|c|}
\hline $\begin{array}{c}\text { Overall } \\
\text { stress }\end{array}$ & $\mathbf{1 5 . 7 1}$ & $\mathbf{1 . 6 1}$ & $\mathbf{1 7 . 8 2}$ & $\begin{array}{c}\mathbf{2 . 4} \\
\mathbf{0}\end{array}$ & $\mathbf{2 . 1 0 9}$ & $\begin{array}{c}\mathbf{0 . 0 3 6} \\
*\end{array}$ \\
\hline Family & 13.56 & 2.35 & 14.29 & $\begin{array}{c}2.9 \\
2\end{array}$ & 2.358 & $\begin{array}{c}0.019 \\
*\end{array}$ \\
\hline School & 18.33 & 2.89 & 18.97 & $\begin{array}{c}3.5 \\
1\end{array}$ & 1.737 & $\begin{array}{c}0.031 \\
*\end{array}$ \\
\hline Peer & 16.89 & 3.65 & 17.44 & $\begin{array}{c}3.2 \\
0\end{array}$ & 0.867 & $\begin{array}{c}0.036 \\
*\end{array}$ \\
\hline $\begin{array}{c}\text { Overall self- } \\
\text { esteem }\end{array}$ & $\mathbf{3 5 . 2 2}$ & $\mathbf{4 . 1 8}$ & $\mathbf{3 6 . 4 1}$ & $\begin{array}{c}\mathbf{4 . 3} \\
\mathbf{5}\end{array}$ & $\mathbf{1 . 2 2 9}$ & $\begin{array}{c}\mathbf{0 . 0 2 2} \\
*\end{array}$ \\
\hline
\end{tabular}

Since P value is less than 0.05 , null hypothesis is rejected at $5 \%$ level with respect to all the above factors of brain-based learning strategies influencing academic motivation, stress and self-esteem among high school students. Hence there is significant difference between age and level of agreement on factors of brain-based learning strategies influencing academic motivation, stress and self-esteem among high school students. Based on mean score, it can be seen that students who are above 16 years of age have indicated a higher level of agreement with respect to all the factors of brain-based learning strategies influencing academic motivation, stress and self-esteem among high school students.

\section{HYPOTHESIS II}

Null Hypothesis: There is no significant difference between annual income of family and level

of agreement on influence of brain-based learning strategies on academic motivation, stress and self-esteem

Table 3 : ANOVA for significant difference between annual family income and level of agreement on influence of brainbased learning strategies on academic motivation, stress and self-esteem

\begin{tabular}{|c|c|c|c|c|c|c|}
\hline \multirow[t]{2}{*}{ Factors } & \multicolumn{4}{|c|}{$\begin{array}{c}\text { Annual Family Income in } \\
\text { Rupees }\end{array}$} & \multirow[b]{2}{*}{$\begin{array}{c}\text { F } \\
\text { valu } \\
\text { e }\end{array}$} & \multirow[b]{2}{*}{$\begin{array}{c}\mathbf{P} \\
\text { value }\end{array}$} \\
\hline & $\begin{array}{l}\text { Belo } \\
\text { W } \\
\text { Rs. } 5 \\
\text { Lakh } \\
\quad \text { S }\end{array}$ & $\begin{array}{l}\text { Rs. } 5 \\
-7.5 \\
\text { Lakh } \\
\text { s }\end{array}$ & $\begin{array}{c}\text { Rs, } \\
7.5- \\
10 \\
\text { Lak } \\
\text { hs }\end{array}$ & $\begin{array}{c}\text { Abov } \\
\text { e Rs. } \\
10 \\
\text { Lakh } \\
\text { s }\end{array}$ & & \\
\hline $\begin{array}{c}\text { Brain } \\
\text { based } \\
\text { learning } \\
\text { strategies }\end{array}$ & $\begin{array}{c}18.43 \\
8 \\
(2.19 \\
)\end{array}$ & $\begin{array}{c}19.62 \\
(3.20 \\
)\end{array}$ & $\begin{array}{c}19.0 \\
8 \\
(2.23 \\
) \\
\end{array}$ & $\begin{array}{l}20.94 \\
(3.97)\end{array}$ & $\begin{array}{c}1.80 \\
6\end{array}$ & $\begin{array}{c}0.452 \\
*\end{array}$ \\
\hline $\begin{array}{l}\text { Learning } \\
\text { value }\end{array}$ & $\begin{array}{c}12.96 \\
(2.21 \\
)\end{array}$ & $\begin{array}{c}12.77 \\
(2.27 \\
)\end{array}$ & $\begin{array}{c}12.7 \\
4 \\
(2.17 \\
) \\
\end{array}$ & $\begin{array}{l}14.20 \\
(2.69)\end{array}$ & $\begin{array}{c}1.85 \\
5\end{array}$ & $\begin{array}{c}0.013 \\
*\end{array}$ \\
\hline $\begin{array}{c}\text { Active } \\
\text { learning } \\
\text { strategies }\end{array}$ & $\begin{array}{c}17.72 \\
(2.70 \\
)\end{array}$ & $\begin{array}{c}15.62 \\
(3.26 \\
)\end{array}$ & $\begin{array}{c}15.6 \\
6 \\
(3.15 \\
) \\
\end{array}$ & $\begin{array}{l}15.86 \\
(3.07)\end{array}$ & $\begin{array}{c}2.36 \\
2\end{array}$ & $\begin{array}{c}0.019 \\
*\end{array}$ \\
\hline $\begin{array}{c}\text { Self- } \\
\text { efficacy }\end{array}$ & $\begin{array}{c}17.48 \\
(2.18 \\
)\end{array}$ & $\begin{array}{c}18.62 \\
(3.30 \\
)\end{array}$ & $\begin{array}{c}18.0 \\
2 \\
(3.23 \\
)\end{array}$ & $\begin{array}{l}18.94 \\
(2.97)\end{array}$ & $\begin{array}{c}1.80 \\
6\end{array}$ & $\begin{array}{c}0.014 \\
*\end{array}$ \\
\hline $\begin{array}{c}\text { Performan } \\
\text { ce goal }\end{array}$ & $\begin{array}{l}12.16 \\
(4.44\end{array}$ & $\begin{array}{l}17.69 \\
(2.84\end{array}$ & $\begin{array}{c}17.4 \\
0\end{array}$ & $\begin{array}{l}17.83 \\
(2.84)\end{array}$ & $\begin{array}{c}0.98 \\
1\end{array}$ & $\begin{array}{c}0.040 \\
*\end{array}$ \\
\hline
\end{tabular}

\begin{tabular}{|c|c|c|c|c|c|c|}
\hline & ） & ） & $\begin{array}{c}(1.42 \\
)\end{array}$ & & & \\
\hline $\begin{array}{l}\text { Achievem } \\
\text { ent goal }\end{array}$ & $\begin{array}{c}21.36 \\
(2.78 \\
)\end{array}$ & $\begin{array}{c}20.41 \\
(4.44 \\
)\end{array}$ & $\begin{array}{c}20.5 \\
7 \\
(3.26 \\
)\end{array}$ & $\begin{array}{l}21.14 \\
(4.66)\end{array}$ & $\begin{array}{c}0.59 \\
7\end{array}$ & $\begin{array}{c}0.017 \\
*\end{array}$ \\
\hline $\begin{array}{c}\text { Learning } \\
\text { environme } \\
\text { nt }\end{array}$ & $\begin{array}{c}12.64 \\
(1.89 \\
)\end{array}$ & $\begin{array}{c}13.12 \\
(2.94 \\
)\end{array}$ & $\begin{array}{c}13.5 \\
3 \\
(2.41 \\
)\end{array}$ & $\begin{array}{l}14.94 \\
(2.75)\end{array}$ & $\begin{array}{c}3.08 \\
9\end{array}$ & $\begin{array}{c}0.030 \\
*\end{array}$ \\
\hline $\begin{array}{c}\text { Overall } \\
\text { student } \\
\text { motivatio } \\
\text { n }\end{array}$ & $\begin{array}{c}14.24 \\
(3.43 \\
)\end{array}$ & $\begin{array}{c}14.99 \\
(2.71 \\
)\end{array}$ & $\begin{array}{c}15.0 \\
9 \\
(2.68 \\
)\end{array}$ & $\begin{array}{l}15.48 \\
(2.69)\end{array}$ & $\begin{array}{c}3.83 \\
6\end{array}$ & $\begin{array}{c}0.013 \\
*\end{array}$ \\
\hline Emotional & $\begin{array}{c}11.24 \\
(5.32 \\
)\end{array}$ & $\begin{array}{c}12.52 \\
(2.37 \\
)\end{array}$ & $\begin{array}{c}10.1 \\
9 \\
(2.48 \\
)\end{array}$ & $\begin{array}{l}13.57 \\
(1.78)\end{array}$ & $\begin{array}{c}1.40 \\
0\end{array}$ & $\begin{array}{c}0.024 \\
*\end{array}$ \\
\hline $\begin{array}{c}\text { Physiologi } \\
\text { c }\end{array}$ & $\begin{array}{c}17.51 \\
(2.80 \\
)\end{array}$ & $\begin{array}{c}13.60 \\
(2.40 \\
)\end{array}$ & $\begin{array}{c}12.3 \\
6 \\
(4.43 \\
) \\
\end{array}$ & $\begin{array}{l}14.61 \\
(2.08)\end{array}$ & $\begin{array}{c}3.23 \\
8\end{array}$ & $\begin{array}{c}0.022 \\
*\end{array}$ \\
\hline Beha & $\begin{array}{c}14.72 \\
(2.03 \\
)\end{array}$ & $\begin{array}{c}16.68 \\
(2.09 \\
)\end{array}$ & $\begin{array}{c}16.0 \\
2 \\
(2.16 \\
)\end{array}$ & $\begin{array}{l}17.10 \\
(2.93)\end{array}$ & $\begin{array}{c}2.69 \\
2\end{array}$ & $\begin{array}{c}0.046 \\
*\end{array}$ \\
\hline Cog & $\begin{array}{c}20.72 \\
(2.33 \\
)\end{array}$ & $\begin{array}{c}21.35 \\
(2.96 \\
)\end{array}$ & $\begin{array}{c}21.4 \\
1 \\
(2.49 \\
)\end{array}$ & $\begin{array}{l}22.41 \\
(2.49)\end{array}$ & $\begin{array}{c}1.49 \\
9\end{array}$ & $\begin{array}{c}0.021 \\
*\end{array}$ \\
\hline $\begin{array}{c}\text { Overall } \\
\text { academic } \\
\text { stress }\end{array}$ & $\begin{array}{c}10.16 \\
(3.21 \\
)\end{array}$ & $\begin{array}{c}12.40 \\
(2.61 \\
)\end{array}$ & $\begin{array}{c}13.6 \\
9 \\
(1.73 \\
) \\
\end{array}$ & $\begin{array}{l}16.03 \\
(2.71)\end{array}$ & $\begin{array}{c}4.31 \\
9\end{array}$ & $\begin{array}{c}\mathbf{0 . 0 1 5} \\
*\end{array}$ \\
\hline Family & $\begin{array}{c}13.16 \\
(1.18 \\
)\end{array}$ & $\begin{array}{c}13.04 \\
(2.74 \\
)\end{array}$ & $\begin{array}{c}13.4 \\
8 \\
(1.83 \\
)\end{array}$ & $\begin{array}{c}14.15 \\
(26.7 \\
9)\end{array}$ & $\begin{array}{c}3.14 \\
5\end{array}$ & $\begin{array}{c}0.025 \\
*\end{array}$ \\
\hline School & $\begin{array}{c}13.72 \\
(2.91 \\
)\end{array}$ & $\begin{array}{c}13.94 \\
(2.97 \\
)\end{array}$ & $\begin{array}{c}14.4 \\
8 \\
(3.15 \\
) \\
\end{array}$ & $\begin{array}{l}14.98 \\
(3.34)\end{array}$ & $\begin{array}{c}0.55 \\
7\end{array}$ & $\begin{array}{c}0.044 \\
*\end{array}$ \\
\hline Peer & $\begin{array}{c}17.32 \\
24.59 \\
)\end{array}$ & $\begin{array}{c}18.81 \\
(3.65 \\
)\end{array}$ & $\begin{array}{c}18.9 \\
8 \\
(3.46 \\
) \\
\end{array}$ & $\begin{array}{l}19.76 \\
(3.57)\end{array}$ & $\begin{array}{c}1.36 \\
0\end{array}$ & $\begin{array}{c}0.025 \\
*\end{array}$ \\
\hline $\begin{array}{c}\text { Overall } \\
\text { student } \\
\text { self- } \\
\text { esteem }\end{array}$ & $\begin{array}{c}24.32 \\
(3.09 \\
)\end{array}$ & $\begin{array}{c}26.87 \\
\mathbf{a} \\
(3.19 \\
) \\
\end{array}$ & $\begin{array}{c}29.1 \\
4 \\
(3.42 \\
) \\
\end{array}$ & $\begin{array}{l}30.55 \\
(3.48)\end{array}$ & $\begin{array}{c}2.88 \\
4\end{array}$ & $\begin{array}{c}\mathbf{0 . 0 3 3} \\
*\end{array}$ \\
\hline
\end{tabular}

Since $\mathrm{P}$ value is less than 0.05 , null hypothesis is rejected at $5 \%$ level with respect to all the above factors of brain-based learning strategies influencing academic motivation, stress and self-esteem among high school students. Hence there is significant difference between annual family income and level of agreement on factors of brain-based learning strategies influencing academic motivation, stress and selfesteem among high school students. High school students who hail from families whose annual income is more than Rs. 10 lakhs have shown a higher level of agreement with 
respect to all the factors of brain-based learning strategies influencing academic motivation, stress and self-esteem

Table 4 : Correlation between influence of brain-based learning strategies on student motivation, stress and self-

\begin{tabular}{|c|c|c|c|c|}
\hline Factors & $\begin{array}{c}\text { Brain- } \\
\text { based } \\
\text { learning } \\
\text { strategies }\end{array}$ & $\begin{array}{c}\text { Student } \\
\text { motivation }\end{array}$ & Stress & $\begin{array}{c}\text { Self- } \\
\text { esteem }\end{array}$ \\
\hline $\begin{array}{c}\text { Brain-based } \\
\text { learning } \\
\text { strategies }\end{array}$ & 1 & $0.728^{* *}$ & $0.714^{* *}$ & $0.725^{* *}$ \\
\hline $\begin{array}{c}\text { Student } \\
\text { motivation }\end{array}$ & & 1 & $0.612^{* *}$ & $0.655^{* *}$ \\
\hline Stress & & & 1 & $0.676^{* *}$ \\
\hline Self-esteem & & & & 1 \\
\hline
\end{tabular}

The above table shows that there is a positive relationship among brain-based learning strategies and its influence on student motivation, stress and self-esteem among high school students. There is also positive correlation among student motivation, stress and self-esteem.

\section{Findings and Conclusion}

The results of the study have shown that there is significant difference between gender, age and annual family income and the level of agreement on factors of brain-based learning strategies influencing academic motivation, stress and selfesteem among high school students. Further analysis has highlighted that female students who are above 16 years of age and who hail from families having an annual income of more than Rs. 10 lakhs have indicated a higher level of agreement with respect to all the factors of brain-based learning strategies influencing academic motivation, stress and self-esteem among high school students.

This study has undoubtedly proved that importance of brainbased learning strategies in improving student motivation, reducing stress and leading to higher self-esteem among high school students which in sure to result in improved academic performance. As teacher are expected to render help to students, by having appropriate experiences and capitalising on such experiences would help them in knowing how the brain works best which in turn would allow teachers to create an environment that gives the student a higher probability of success in learning.

\section{References}

[1] Caine, G.; Nummela-Caine, R. (1994). Making Connections: Teaching and the Human Brain. Menlo Park, CA.: AddisonWesley Longman, Incorporated. ISBN: 978-0201490886

[2] Hasra, K. (2007). Brain development, structuring of learning and science education: Where are we now? A review of some recent research. International Journal of Science Education, 24(4), 343356.

[3] Keleş, E. ve Çepni, S. (2006). Beyin ve öğrenme. Türk Fen Eğitimi Dergisi, 3(2), 66-82.

[4] Materna (2000). The impact of brain-based instruction on reading achievement in a second grade classroom (Doctoral dissertation, Walden University, College of Education, Minnesota).

[5] science among secondary school students in relation to their scientific creativity, 2019,

[6] Sousa, D. (1998-2011) How the Brain Learns (with learning manual). Thousand Oaks, CA: Corwin Press Incorporated.

[7] Weiss, R. P. (2000). Brain-based learning: The wave of the brain. Training \& Development, July, 20-24. Retrieved from

[8] Zaudeh and Al Astal (2014). Effect of Brain Based Learning Strategy on life skills and achievement in 